\title{
INCIDÊNCIA DE PLANTAS ESPONTÂNEAS EM CULTIVARES DE PIMENTÃO, PRODUZIDAS EM DIFERENTES COBERTURAS MORTAS
}

\author{
Francisco Teixeira Matos Junior'; Franciely da Silva Ponce²; Anna karoliny Oliveira Lima'; Núbia de Fátima \\ Alves Santos 3; Márcio Roberto da Silva Melo ${ }^{3}$; Vitor Quintela de Sousa'; Luciana da Silva Borges33; Luis de \\ Souza Freitas ${ }^{3}$.
${ }^{1}$ Graduandos em Agronomia da Universidade Federal Rural da Amazônia (UFRA), Paragominas, Pará, Brasil, matos.junior01@gmail.com, oliverannakarol@gmail.com, quintelav@gmail.com
Eng. Agr. MSc. UNEMAT, Campus Nova Mutum, Maranhão, Brasil, francyponce@hotmail.com
3Professor (a) Dr. (a) da UFRA, Paragominas, Pará, Brasil, nubia.santos@ufra.edu.br, marcio.melo@ufra.edu.br, luciana.borges@ufra.edu.br, luis.freitas@ufra.edu.br

RESUMO: A utilização de cobertura morta tem se mostrado uma alternativa no cultivo de hortaliças uma vez que possibilita a redução da oscilação da temperatura do solo, evaporação de água do mesmo, perda de adubos e corretivos por lixiviação. Desta forma objetivou se avaliar a incidência das plantas espontâneas em cultivo de pimentão sob diferentes coberturas mortas. $O$ experimento foi conduzido na Universidade Federal Rural da Amazônia, campus de Paragominas, na área experimental de Horticultura. 0 delineamento estatístico utilizado foi em esquema fatorial $3 \times 4$, sendo utilizadas três cultivares de pimentão: Ikeda, Rubi Gigante e Quadrado vermelho. As coberturas mortas utilizadas foram: palha de arroz, caroço de açái, resíduo de soja e a testemunha. As quais, foram distribuídas de maneira uniforme nas parcelas, com $5 \mathrm{~cm}$ de camada de cada material. Avaliou - se a incidência de plantas daninhas na produção de pimentão, utilizando se o método de levantamento do tipo quadrado inventariado, com o auxílio de moldura de madeira, com área interna de 0,50 $\mathrm{m}^{2}$, colocado no centro de cada parcela. A cobertura morta com caroço de açaíapresentou melhor tendência no controle de plantas daninhas na cv. Rubi Gigante e na cv. Casca Dura Ikeda de pimentão. Enquanto que o resíduo de soja apresentou tendência a ser mais eficiente no cultivo de pimentão cV. Quadrado Vermelho.

PALAVRAS-CHAVE: Cobertura do solo, Hortaliça-fruto, Palha de arroz.

\section{INCIDENCE OF SPONTANEOUS PLANTS IN CULTIVARS OF CHILI PEPPER, PRODUCED IN DIFFERENT DEAD COVERINGS}

\begin{abstract}
The use of mulch has shown to be an alternative in the cultivation of vegetables since it allows the reduction of soil temperature oscillation, evaporation of soil water, loss of fertilizers and correctives by leaching. The objective of this study was to evaluate the incidence of spontaneous plants in sweet pepper under different coverages. The experiment was conducted at the Federal Rural University of Amazonia, Campus Paragominas, in the experimental area of Horticulture. The statistical design was used in a $3 \times 4$ factorial scheme, using three chili cultivars: Ikeda, Rubi Gigante and Red square. The
\end{abstract}


dead coverages used were: rice straw, sugarcane, soybean residue and the control. They were distributed evenly in the plots, with $5 \mathrm{~cm}$ of layer of each material. We evaluated the incidence of weeds in the production of sweet pepper, using the method of survey of the square type inventoried, with the aid of a wooden frame, with an internal area of $0.50 \mathrm{~m}^{2}$, placed in the center of each plot. The dead cover with açaí stone presented a better trend in the control of weeds in cv. Ruby Giant and in cv. Ikeda hard shell of chili. While the soybean residue tended to be more efficient in the cultivation of cv. Red Square.

KEYWORDS: Fruit vegetable, Rice straw, Soil cover.

\section{INCIDENCIA DE PLANTAS ESPONTÁNEAS EN CULTIVARES DE PIMIENTO, PRODUCCIONES EN DIFERENTES CUBIERTAS MUERTAS}

RESUMEN: La utilización de cobertura muerta se ha mostrado una alternativa en el cultivo de hortalizas ya que posibilita la reducción de la oscilación de la temperatura del suelo, evaporación de agua del mismo, pérdida de abonos y correctivos por lixiviación. De esta forma se objetivó evaluar la incidencia de las plantas espontáneas en cultivo de pimiento bajo diferentes coberturas muertas. El experimento fue conducido en la Universidad Federal Rural de la Amazonia, campus de Paragominas, en el área experimental de Horticultura. El delineamiento estadístico utilizado fue en esquema factorial $3 \times 4$, siendo utilizadas tres cultivares de pimiento: Ikeda, Rubí Gigante y Cuadrado rojo. Las coberturas muertas utilizadas fueron: paja de arroz, hueso de azái, residuo de soja y el testigo. Las cuales, fueron distribuidas de manera uniforme en las parcelas, con $5 \mathrm{~cm}$ de capa de cada material. Se evaluó la incidencia de plantas dañinas en la producción de pimiento, utilizando el método de levantamiento del tipo cuadrado inventariado, con el auxilio de marco de madera, con área interna de 0,50 $\mathrm{m}^{2}$, colocado en el centro de cada parcela. La cobertura muerta con el hueso de azaí en la mejor tendencia en el control de las plantas dañinas en la cv. Rubi Gigante y en la cv. Casca Dura Ikeda de chile. Mientras que el residuo de soja presentó tendencia a ser más eficiente en el cultivo de pimiento cv. Cuadrado Rojo.

PALABRAS CLAVE: Cobertura del suelo, Hortalizas, Paja de arroz.

O pimentão (Capsicum annum L.) pertence à família das Solanaceas e é originário das Américas. Está entre as dez hortaliças mais consumidas no país sendo uma das mais importantes em relação ao valor e o volume comercializado (ECHER et al., 2002; ALBUQUERQUE et al., 2011). Apesar de ser uma espécie adaptada a climas tropicais o cultivo do pimentão 
apresenta alto custo e requer um alto nível tecnológico do produtor (COELHO, 2011).

Segundo Coelho et al., (2013), o pimentão é muito sensível à interferência das plantas espontâneas, uma vez que o seu crescimento inicial é lento em relação às plantas iespontâneas e também um menor índice de área foliar, com isso é importante a realização do controle das plantas espontâneas em todo o ciclo da cultura. Para os autores Gomes e Christoffoleti (2008) O controle de plantas espontâneas é importante o uso de estratégias que interfiram no desenvolvimento das mesmas e consequentemente contribuam para um melhor desempenho da cultura principal, nesse caso, do pimentão. Logo o uso de cobertura morta irá contribuir com a inteferir no desenvolvimento das plantas espontâneas e favorecerá a cultura pincipal.

A utilização de cobertura morta tem se mostrado uma alternativa no cultivo de hortaliças uma vez que possibilita a redução da oscilação da temperatura do solo, evaporação de água do mesmo, perda de adubos e corretivos por lixiviação, minimizando assim, a compactação, erosão, e o contato direto dos frutos com o solo, melhorando a aparência visual dos mesmos (BRAGA et al., 2010). Além disso, sua utilização atua como bloqueador de luz solar, o que acaba dificultando a quebra de dormência das sementes de plantas espontâneas. 0 que permiti a diminuição da infestação por estas plantas, sem necessidade de aplicação de produtos químicos, resultando em conservação do solo e microbiota, e consequente, diminuição de gastos com herbicidas.

Estudos relacionados com avaliação de cultivares adaptadas as condições edafoclimáticas de Paragominas são escassos ou não publicados. $\bigcirc$ que pode trazer avanços para produção destas espécies em regiões tropicais na recomendação de materiais que têm melhor potenciais. Contribuindo com suporte técnico para implantar boas práticas de manejo e inserir novas cultivares no Estado do Pará. E efetivar a transferência de conhecimentos e tecnologias para fomento da olericultura paraense.

Diversos materiais podem ser utilizados como coberturas de solo, variando desde 
o uso de mulching, plantas dissecadas como é utilizado no sistema plantio direto ou mesmo pode ser utilizados resíduos tais como bagaço de cana e palha de arroz, ou materiais disponíveis. Desta forma objetivou se avaliar a incidência das plantas espontâneas em cultivo de pimentão sob diferentes coberturas mortas.

O experimento foi conduzido na Universidade Federal Rural da Amazônia, campus de Paragominas, na área experimental de Horticultura. De fevereiro de 2017 e junho de 2017. O município de Paragominas está situado nas seguintes coordenadas geográficas $02^{\circ} 55^{\prime} 24^{\prime \prime} \mathrm{S}$ e $47^{\circ} 34^{\prime} 36^{\prime \prime}$ W. O solo da área experimental é do tipo Latossolo Amarelo muito argiloso (EMBRAPA, 1999). Sendo o clima da região tropical chuvoso, do tipo Aw, segundo a classificação de Köppen, com estação seca bem definida e com temperatura média anual de $26,5^{\circ} \mathrm{C}$.

Foi realizada a limpeza da área, para a retirada das plantas invasoras existentes no local. Realizou-se analise do solo, onde os resultados indicaram: $P\left(\mathrm{mg} / \mathrm{dm}^{3}\right)=32$; M.O $\left(\mathrm{g} / \mathrm{dm}^{3}\right)=22 ; \mathrm{pH}(\mathrm{CaCl} 2)=4,9 ; \mathrm{K}$ $\left(\mathrm{mmolc} / \mathrm{dm}^{3}\right)=1,9 ; \mathrm{Ca}\left(\mathrm{mmolc} / \mathrm{dm}^{3}\right)=12 ;$ $\operatorname{Mg}\left(\mathrm{mmolc} / \mathrm{dm}^{3}\right)=6 ; \mathrm{H}^{\circ}+\mathrm{Al}^{3}$ $\left(\mathrm{mmolc} / \mathrm{dm}^{3}\right)=42 ; H^{\circ}\left(\mathrm{mmolc} / \mathrm{dm}^{3}\right)=37 ;$

C.T.C. $\left(\mathrm{mmolc} / \mathrm{dm}^{3}\right)=47,5 ;$ S.B. $\left(\mathrm{mmolc} / \mathrm{dm}^{3}\right)=18,6 ; \mathrm{S}\left(\mathrm{mg} / \mathrm{dm}^{3}\right)=35 ; \mathrm{V} \%$ $\left(\mathrm{mg} / \mathrm{dm}^{3}\right)=23 ; \mathrm{K}$ na CTC $(\%)=2,9 ;$ Ca na CTC $(\%)=26,8 ; \mathrm{Mg}$ na CTC $(\%)=4,8 ; \mathrm{Al}$ na CTC (\%) =7,8; H na CTC (\%) = 40,7; $m$ $(\%)=12,56 ; \mathrm{Ca} / \mathrm{K}(\%)=7,4 ; \mathrm{Ca} / \mathrm{M}(\%)=$ 6,$8 ; \mathrm{Mg} / \mathrm{K}(\%)=2,0$. A partir dos resultados da análise de solo, foi realizada a adubação dos canteiros, usando como mistura: terra preta e fertilizante químico (NPK, 10-28-20) na medida de $100 \mathrm{~g} / \mathrm{m}^{2}$ e para a correção do pH, $100 \mathrm{~g} / \mathrm{m}^{2}$ de calcário dolomítico. Foi realizado o revolvimento do solo e posteriormente o levantamento dos canteiros que possuíam 1,2 m de largura e $20 \mathrm{~cm}$ de altura.

As mudas de pimentão foram produzidas em bandejas de poliestireno expandido de 128 células, preenchidas com areia preta peneirada em janeiro de 2017. As bandejas foram colocadas em viveiro, com dimensões de 4x12 metros, pé direito de 3 metros e coberto com sombrite $70 \%$. O delineamento estatístico utilizado foi em esquema fatorial $3 \times 4$, sendo utilizadas três cultivares de pimentão sendo elas: Ikeda, Rubi Gigante e Quadrado vermelho. As coberturas mortas 
utilizadas foram: palha de arroz, caroço de açái, resíduo de soja e a testemunha. A palha de arroz e o resíduo de soja, foram obtidos das empresas de grãos de Paragominas, e o caroço de açaí, foi obtido dos comerciantes que processam o açaí na região de Paragominas, esses materiais ficam cerca 6 meses em processo de composição na área da UFRA. Esses materias foram distribuídos de maneira uniforme nas parcelas, com $5 \mathrm{~cm}$ de camada de cada material.

transplante foi realizado 30 dias após a semeadura, quando as plântulas apresentaram de quatro a seis folhas definitivas. $\bigcirc$ espaçamento utilizado foi de $40 \mathrm{~cm}$ entre plantas e $70 \mathrm{~cm}$ entre linhas. Utilizou se irrigação por aspersão, com mangueiras do tipo Santeno, sendo realizadas duas vezes ao dia.

Durante a condução do experimento avaliou se a incidência de plantas daninhas na produção de pimentão, utilizando se o método de levantamento do tipo quadrado inventariado, com o auxílio de moldura de madeira, com área interna de 0,50 $\mathrm{m}^{2}$, colocado no centro de cada parcela. As plantas daninhas foram coletadas, separadas e identificadas segundo Lorenzi (2006).

Utilizou-se o programa Microsoft Office Excel para realização de análise descritiva e obtenção de tabelas e análise.

Foram identificadas 28 espécies de plantas espontaneas, na cultura do pimentão cv. Rubi Gigante, nas diferentes coberturas mortas, distribuídas em cinco famílias, como pode ser observado na Tabela 1. A família mais representativa no que se refere a número de espécies, foi a Cyperaceae.

Verifica-se na Tabela 1, que houve uma tendência a cobertura morta com caroço de açaí apresentar menor incidência de plantas espontâneas, no cultivo de pimentão, cv. Rubi Gigante, apresentando um total de indivíduos de 251, na sequência, resíduo de soja com 258, e palha de arroz com 331 indivíduos no total.

Cyperus iria L. foi a espécie com maior incidência, em todas as coberturas mortas utilizanda na cv. Rubi Gigante, apresentando 242 individuos na cobertura com resíduo de soja, 221, no caroço de açaí, 323 na palha de arroz e na testemunha 285 individuos. Segundo Pereira e Melo (2008), a incidência de 
tiririca na área de cultivo, pode ser um indicativo de solo ácido e com carência de magnésio. A análise química do solo da área do experimento, apresentou $\mathrm{pH}$ $\left(\mathrm{CaCl}_{2}\right)=4,9$, ou seja, $\mathrm{pH}$ ácido, o que provavelmente contribuiu para esse quantitativo de tiririca.

Na tabela 2, verifica-se a identificação de 38 espécies de plantas espontâneas, na cultura do pimentão cv. Casca Dura Ikeda, nas diferentes coberturas mortas, distribuídas em nove famílias. Onde a família mais representativa no que se refere a número de espécies, foi a Cyperaceae.

A cobertura morta que apresentou uma tendência a menor mais eficaz incidência de plantas espontâneas no cultivo de pimentão cv. Casca Dura Ikeda, foi com caroço de açaí, com 328 indivíduos total, seguida da palha de arroz, com 301, resíduo de soja com 338 indivíduos total (Tabela 2). A cobertura morta é capaz de alterar umidade, luminosidade e temperatura superficial do solo, dificultando a quebra de dormência e a germinação de sementes, além de se constituir em barreira mecânica (CONSTANTIN, 2001). Sendo uma alternativa no controle de plantas espontâneas para pequenos produtores de hortaliças.

Com relação a tabela 3, verifica-se a identifição de 42 espécies de plantas espontâneas, na cultura do pimentão cv. Quadrado Vermelho, nas diferentes coberturas mortas, distribuídas em 12 famílias. Onde a família mais representativa no que se refere a número de espécies, foi a Cyperaceae.

Constata-se na Tabela 3, que no cultivo de pimentão cv. Quadrado vermelho, a cobertura morta, que apresentou tendência a diminuir a incidência de plantas espontâneas, foi com Resíduo de soja, onde foi contabilizado 155 indivíduos no total, enquanto que no caroço de açaí foi um total de 313 indivíduos, e palha de arroz com 317 indivíduos total. Outros autores têm evidenciado o potencial de controle de ervas espontâneas através da adição de resíduos de leguminosas (ERASMO et al., 2004) à superfície do solo. Esses resultados são geralmente associados a efeitos físicos e químicos.

A planta espontânea que obteve maior frequência em todas as coberturas mortas, nas três cultivares de pimentão 
foi a Cyperus rotundus L. (Cyperaceae), conhecida no Brasil como tiririca, que é considerada a planta daninha mais disseminada e agressiva de todo o mundo (CUDNEY, 1997; DURIGAN et al., 2005). Em muitos agroecossistemas é considerada como a principal planta daninha (BLANCO, 2006), provocando reduções quantitativas e qualitativas na produção mundial das principais culturas (CUDNEY, 1997).

A cobertura morta com caroço de açaíapresentou melhor tendência no controle de plantas daninhas na cv. Rubi Gigante e na cv. Casca Dura Ikeda de pimentão. Enquanto que o resíduo de soja apresentou tendência a ser mais eficiente no cultivo de pimentão cv. Quadrado Vermelho.

Tabela 1. Incidência de plantas daninhas no cultivo de pimentão Rubi Gigante sob diferentes coberturas mortas, no Município de Paragominas- Pa

\begin{tabular}{|c|c|c|c|c|c|}
\hline Tratamentos & Nome vulgar & Família botânica & Nome científico & $\begin{array}{l}\text { No. } \\
\text { Indivíduos }\end{array}$ & $\begin{array}{c}\text { Total de } \\
\text { individuos/ } \\
\mathrm{m}^{2}\end{array}$ \\
\hline \multirow{7}{*}{ Resíduo de Soja } & Tiririca & Cyperaceae & Cyperus iria L. & 242 & \multirow{7}{*}{258} \\
\hline & Brachiaria & Poaceae & $\begin{array}{c}\text { Brachiaria decumbens } \\
\text { Stapf cv. Comum }\end{array}$ & 6 & \\
\hline & Carurú & Amaranthaceae & Amaranthus deflexus L. & 2 & \\
\hline & Grama seda & Poaceae & Cynodon dactylon & 2 & \\
\hline & $\begin{array}{l}\text { Capim } \\
\text { Colchão }\end{array}$ & Poaceae & $\begin{array}{l}\text { Digitaria horinzontalis } \\
\text { Willd. }\end{array}$ & 2 & \\
\hline & Pé de galinha & Poaceae & Eleusine indica $\mathrm{L}$. & 3 & \\
\hline & Sojinha & Brassicaceae & Cleone affinis DC. & 1 & \\
\hline \multirow{6}{*}{ 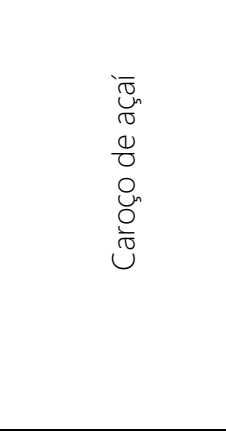 } & Carurú & Amaranthaceae & Amaranthus deflexus L. & 2 & \multirow{6}{*}{251} \\
\hline & $\begin{array}{l}\text { Capim } \\
\text { Colchão }\end{array}$ & Poaceae & $\begin{array}{c}\text { Digitaria horinzontalis } \\
\text { Willd. }\end{array}$ & 6 & \\
\hline & $\begin{array}{l}\text { Quebra- } \\
\text { pedra }\end{array}$ & Euphorbiaceae & Phyllanthus tenellus & 3 & \\
\hline & Tiririca & Cyperaceae & Cyperus iria L. & 221 & \\
\hline & Apaga-fogo & Amaranthaceae & $\begin{array}{c}\text { Alternanthera tenella } \\
\text { Collla. }\end{array}$ & 14 & \\
\hline & Brachiaria & Poaceae & $\begin{array}{l}\text { Brachiariadecumbens } \\
\text { Stapf cv comum }\end{array}$ & 5 & \\
\hline \multirow{3}{*}{$\begin{array}{ll}\frac{0}{0} & N \\
0 & 0 \\
\frac{0}{\sigma} & \frac{0}{\sigma} \\
\frac{0}{\sigma} & \end{array}$} & Tiririca & Cyperaceae & Cyperus iria L. & 323 & \multirow{3}{*}{331} \\
\hline & Carurú & Amaranthaceae & Amaranthus deflexus L. & 1 & \\
\hline & Pé de galinha & Poaceae & Eleusine indica L. & 3 & \\
\hline
\end{tabular}




\begin{tabular}{|c|c|c|c|c|c|}
\hline & $\begin{array}{l}\text { Capim } \\
\text { Colchão }\end{array}$ & Poaceae & $\begin{array}{c}\text { Digitaria horinzontalis } \\
\text { Willd. }\end{array}$ & 4 & \\
\hline \multirow{11}{*}{ 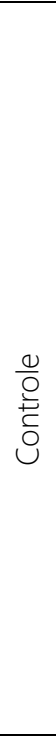 } & Agriãozinho & Brassicaceae & $\begin{array}{c}\text { Cardamine } \\
\text { bonariensis Pers }\end{array}$ & 1 & \\
\hline & Beldroega & Euphorbiaceae & Chamaesyce prostrata A & 1 & \\
\hline & $\begin{array}{l}\text { Botão de } \\
\text { ouro }\end{array}$ & Asteraceae & Unxia kubitzkii & 1 & \\
\hline & Burra leiteira & Euphorbiaceae & Sapium sceleratum & 1 & \\
\hline & Carurú & Amaranthaceae & Amaranthus deflexus L. & 6 & \\
\hline & Tiririca & Cyperaceae & Cyperus iria L. & 285 & \multirow{6}{*}{369} \\
\hline & Sojinha & Brassicaceae & Cleone affinis DC. & 2 & \\
\hline & $\begin{array}{l}\text { Capim } \\
\text { Colchão }\end{array}$ & Poaceae & $\begin{array}{l}\text { Digitaria horinzontalis } \\
\text { Willd. }\end{array}$ & 47 & \\
\hline & Pé de galinha & Poaceae & Eleusine indica $\mathrm{L}$. & 10 & \\
\hline & $\begin{array}{l}\text { Quebra- } \\
\text { pedra }\end{array}$ & Euphorbiaceae & Phyllanthus tenellus & 11 & \\
\hline & Apaga-fogo & Amaranthaceae & $\begin{array}{l}\text { Alternanthera tenella } \\
\text { Collla. }\end{array}$ & 4 & \\
\hline
\end{tabular}

Tabela 2. Incidência de plantas espontâneas no cultivo de pimentão Casca Dura Ikeda sob diferentes coberturas mortas, no Município de Paragominas- Pa.

\begin{tabular}{|c|c|c|c|c|c|}
\hline Tratamentos & Nome vulgar & Família botânica & Nome científico & $\begin{array}{l}\text { No. } \\
\text { Indivíduos }\end{array}$ & $\begin{array}{c}\text { Total de } \\
\text { indivíduos/ } \\
\mathrm{m}^{2} \\
\end{array}$ \\
\hline \multirow{9}{*}{ 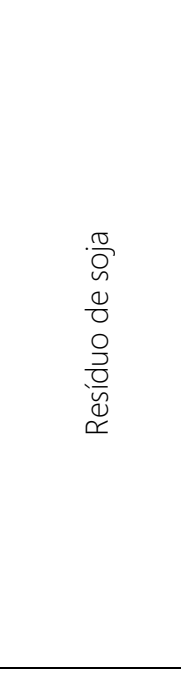 } & Tiririca & Cyperaceae & Cyperus iria L. & 309 & \multirow{9}{*}{338} \\
\hline & Pé de galinha & Poaceae & Eleusine indica $\mathrm{L}$. & 7 & \\
\hline & Quebra-pedra & Euphorbiaceae & Phyllanthus tenellus & 2 & \\
\hline & Carurú & Amaranthaceae & Amaranthus deflexus L. & 8 & \\
\hline & $\begin{array}{l}\text { Capim } \\
\text { Colchão }\end{array}$ & Poaceae & $\begin{array}{l}\text { Digitaria horinzontalis } \\
\text { Willd. }\end{array}$ & 11 & \\
\hline & Apaga-fogo & Amaranthaceae & $\begin{array}{l}\text { Alternanthera tenella } \\
\text { Collla. }\end{array}$ & 1 & \\
\hline & $\begin{array}{l}\text { Capim } \\
\text { colonião }\end{array}$ & Gramineae & $\begin{array}{l}\text { Panicum maximum } \\
\text { Jacq. (PANMA) }\end{array}$ & 1 & \\
\hline & Falsa Tiririca & Hypoxidaceae & Hypoxis decumbens L. & 4 & \\
\hline & Sojinha & Brassicaceae & Cleone affinis DC. & 3 & \\
\hline \multirow{4}{*}{ 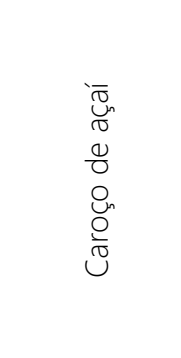 } & Carurú & Amaranthaceae & Amaranthus deflexus L. & 3 & \multirow{4}{*}{328} \\
\hline & Sojinha & Brassicaceae & Cleone affinis DC. & 1 & \\
\hline & $\begin{array}{l}\text { Capim } \\
\text { Colchão }\end{array}$ & Poaceae & $\begin{array}{l}\text { Digitaria horinzontalis } \\
\text { Willd. }\end{array}$ & 8 & \\
\hline & $\begin{array}{l}\text { Capim } \\
\text { colonião }\end{array}$ & Gramineae & $\begin{array}{l}\text { Panicum maximum } \\
\text { Jacq. (PANMA) }\end{array}$ & 2 & \\
\hline
\end{tabular}




\begin{tabular}{|c|c|c|c|c|c|}
\hline & & & & & \\
\hline & Pé de galinha & Poaceae & Eleusine indica $\mathrm{L}$. & 1 & \\
\hline & Quebra-pedra & Euphorbiaceae & Phyllanthus tenellus & 3 & \\
\hline & Brachiaria & Poaceae & $\begin{array}{l}\text { Brachiariadecumbens } \\
\text { Stapf cv Comum }\end{array}$ & 1 & \\
\hline & Tiririca & Cyperaceae & Cyperus iria L. & 291 & \\
\hline & Apaga-fogo & Amaranthaceae & $\begin{array}{c}\text { Alternanthera tenella } \\
\text { Collla. }\end{array}$ & 18 & \\
\hline \multirow{8}{*}{ Palha de arroz } & Tiririca & Cyperaceae & Cyperus iria L. & 263 & \multirow{8}{*}{301} \\
\hline & Falsa Tiririca & Hypoxidaceae & Hypoxis decumbens L. & 12 & \\
\hline & Falsa serralha & Asteraceae & $\begin{array}{c}\text { Emilia fosbergii } \\
\text { Nicolson. }\end{array}$ & 3 & \\
\hline & Sojinha & Brassicaceae & Cleone affinis DC. & 3 & \\
\hline & Pé de galinha & Poaceae & Eleusine indica L. & 4 & \\
\hline & Carurú & Amaranthaceae & Amaranthus deflexus L. & 1 & \\
\hline & $\begin{array}{l}\text { Capim } \\
\text { Colchão }\end{array}$ & Poaceae & $\begin{array}{c}\text { Digitaria horinzontalis } \\
\text { Willd. }\end{array}$ & 11 & \\
\hline & Apaga-fogo & Amaranthaceae & $\begin{array}{l}\text { Alternanthera tenella } \\
\text { Collla. }\end{array}$ & 4 & \\
\hline \multirow{12}{*}{$\begin{array}{l}\frac{0}{0} \\
\stackrel{0}{0} \\
0\end{array}$} & Tiririca & Cyperaceae & Cyperus iria L. & 520 & \multirow{12}{*}{652} \\
\hline & Falsa Tiririca & Hypoxidaceae & Hypoxis decumbens L. & 28 & \\
\hline & Sojinha & Brassicaceae & Cleone affinis DC. & 4 & \\
\hline & $\begin{array}{l}\text { Capim } \\
\text { Colchão }\end{array}$ & Poaceae & $\begin{array}{c}\text { Digitaria horinzontalis } \\
\text { Willd. }\end{array}$ & 51 & \\
\hline & Pé de galinha & Poaceae & Eleusine indica $\mathrm{L}$. & 22 & \\
\hline & Lantana & Verbenaceae & Lantana camara & 7 & \\
\hline & Grama seda & Poaceae & Cynodon dactylon & 2 & \\
\hline & Agriãozinho & Brassicaceae & $\begin{array}{c}\text { Cardamine } \\
\text { bonariensis Pers }\end{array}$ & 3 & \\
\hline & Brachiaria & Poaceae & $\begin{array}{l}\text { Brachiaria decumbens } \\
\text { Stapf cv Comum }\end{array}$ & 8 & \\
\hline & Quebra-pedra & Euphorbiaceae & Phyllanthus tenellus & 3 & \\
\hline & Carurú & Amaranthaceae & Amaranthus deflexus L. & 1 & \\
\hline & Apaga-fogo & Amaranthaceae & $\begin{array}{c}\text { Alternanthera tenella } \\
\text { Collla. }\end{array}$ & 3 & \\
\hline
\end{tabular}


Tabela 3. Incidência de plantas espontâneas no cultivo de pimentão Quadrado Vermelho sob diferentes coberturas mortas, no Município de Paragominas- Pa.

\begin{tabular}{|c|c|c|c|c|c|}
\hline Tratamentos & Nome vulgar & Família botânica & Nome científico & $\begin{array}{l}\text { No. } \\
\text { Indivíduos }\end{array}$ & $\begin{array}{c}\text { Total de } \\
\text { indivíduos/ } \\
\mathrm{m}^{2}\end{array}$ \\
\hline \multirow{10}{*}{ 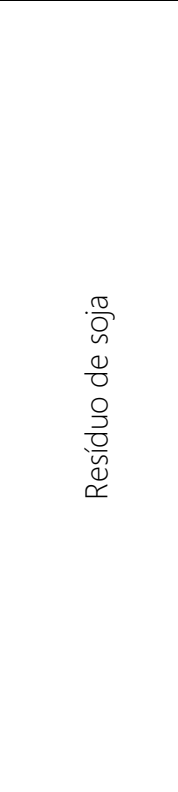 } & Tiririca & Cyperaceae & Cyperus iria L. & 111 & \multirow{10}{*}{155} \\
\hline & Quebra-pedra & Euphorbiaceae & Phyllanthus tenellus & 2 & \\
\hline & Pé de galinha & Poaceae & Eleusine indica $L$. & 11 & \\
\hline & Grama seda & Poaceae & Cynodon dactylon & 7 & \\
\hline & Capim colonião & Gramineae & $\begin{array}{l}\text { Panicum maximum } \\
\text { Jacq. (PANMA) }\end{array}$ & 2 & \\
\hline & Capim Colchão & Poaceae & $\begin{array}{c}\text { Digitaria horinzontalis } \\
\text { Willd. }\end{array}$ & 12 & \\
\hline & Brachiaria & Poaceae & $\begin{array}{l}\text { Brachiaria decumbens } \\
\text { Stapf cv Comum }\end{array}$ & 2 & \\
\hline & Agriãozinho & Brassicaceae & $\begin{array}{c}\text { Cardamine } \\
\text { bonariensis Pers }\end{array}$ & 1 & \\
\hline & Carurú & Amaranthaceae & Amaranthus deflexus L. & 5 & \\
\hline & Beldroega & Portulacaceae & Portulaca oleracea L. & 2 & \\
\hline \multirow{11}{*}{ 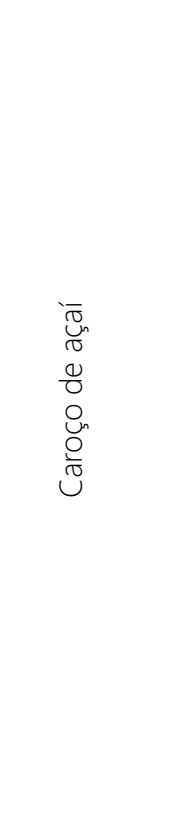 } & Caruru & Amaranthaceae & Amaranthus deflexus L. & 6 & \multirow{11}{*}{313} \\
\hline & Pé de galinha & Poaceae & Eleusine indica $\mathrm{L}$. & 2 & \\
\hline & Capim Colchão & Poaceae & $\begin{array}{c}\text { Digitaria horinzontalis } \\
\text { Willd. }\end{array}$ & 12 & \\
\hline & Sojinha & Brassicaceae & Cleone affinis DC. & 6 & \\
\hline & Quebra-pedra & Euphorbiaceae & Phyllanthus tenellus & 4 & \\
\hline & Tiririca & Cyperaceae & Cyperus iria L. & 265 & \\
\hline & Brachiaria & Poaceae & $\begin{array}{l}\text { Brachiariadecumbens } \\
\text { Stapf cv Comum }\end{array}$ & 1 & \\
\hline & Dormideira & Fabaceae & Mimosa pudica & 1 & \\
\hline & Falsa Tiririca & Hypoxidaceae & Hypoxis decumbens L. & 1 & \\
\hline & Capim colonião & Gramineae & $\begin{array}{l}\text { Panicum maximum } \\
\text { Jacq. (PANMA) }\end{array}$ & 1 & \\
\hline & Apaga-fogo & Amaranthaceae & $\begin{array}{l}\text { Alternanthera tenella } \\
\text { Collla. }\end{array}$ & 14 & \\
\hline \multirow{6}{*}{$\begin{array}{l}N \\
0 \\
\frac{N}{\sigma} \\
0 \\
0 \\
0 \\
0 \\
\frac{D}{\sigma} \\
\bar{\sigma}\end{array}$} & Tiririca & Cyperaceae & Cyperus iria L. & 296 & \multirow{6}{*}{317} \\
\hline & Apaga-fogo & Amaranthaceae & $\begin{array}{l}\text { Alternanthera tenella } \\
\text { Collla. }\end{array}$ & 1 & \\
\hline & Capim amargoso & Gramíneas & Elionurus candidus & 1 & \\
\hline & Burra leiteira & Euphorbiaceae & Sapium sceleratum & 1 & \\
\hline & Caruru & Amaranthaceae & Amaranthus deflexus L. & 1 & \\
\hline & Capim Colchão & Poaceae & $\begin{array}{c}\text { Digitaria horinzontalis } \\
\text { Willd. }\end{array}$ & 11 & \\
\hline
\end{tabular}




\begin{tabular}{|c|c|c|c|c|c|}
\hline & $\begin{array}{c}\text { Sojinha } \\
\text { Pé de galinha }\end{array}$ & $\begin{array}{c}\text { Brassicaceae } \\
\text { Poaceae }\end{array}$ & $\begin{array}{l}\text { Cleone affinis DC. } \\
\text { Eleusine indica L. }\end{array}$ & $\begin{array}{l}2 \\
4 \\
\end{array}$ & \\
\hline \multirow{13}{*}{ 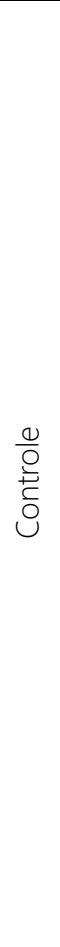 } & Tiririca & Cyperaceae & Cyperus iria L. & 442 & \multirow{13}{*}{562} \\
\hline & Sojinha & Brassicaceae & Cleone affinis DC. & 4 & \\
\hline & Falsa Tiririca & Hypoxidaceae & Hypoxis decumbens L. & 36 & \\
\hline & Beldroega & Portulacaceae & Portulaca oleracea L. & 3 & \\
\hline & Lantana & Verbenaceae & Lantana camara & 3 & \\
\hline & Falsa serralha & Asteraceae & $\begin{array}{l}\text { Emilia fosbergii } \\
\text { Nicolson. }\end{array}$ & 2 & \\
\hline & Capim arroz & Poaceae & $\begin{array}{c}\text { Echinochloa crus-galli } \\
\text { var. crus-galli }\end{array}$ & 6 & \\
\hline & Burra leiteira & Euphorbiaceae & Sapium sceleratum & 1 & \\
\hline & Brachiaria & Poaceae & $\begin{array}{l}\text { Brachiariadecumbens } \\
\text { Stapf cv Comum }\end{array}$ & 1 & \\
\hline & Caruru & Amaranthaceae & Amaranthus deflexus L. & 3 & \\
\hline & Capim Colchão & Poaceae & $\begin{array}{c}\text { Digitaria horinzontalis } \\
\text { Willd. }\end{array}$ & 49 & \\
\hline & Pé de galinha & Poaceae & Eleusine indica L. & 6 & \\
\hline & Apaga-fogo & Amaranthaceae & $\begin{array}{l}\text { Alternanthera tenella } \\
\text { Collla. }\end{array}$ & 6 & \\
\hline
\end{tabular}

\section{REFERÊNCIAS}

ALBUQUERQUE, F. S.; SILVA, E.F.F.; ALBUQUERQUE FILHO, J.A.C.; NUNES, M.F.F.N. Crescimento e rendimento de pimentão fertigado sob diferentes lâminas de irrigação e doses de potássio. Revista Brasileira de Engenharia Agrícola e Ambiental, v. 15, p. 686-694, 2011.

BLANCO, F.M.G. Tubérculo Invasor. Caderno Técnico Cultivar, n.90, p.2-7, 2006.

BRAGA，MB; RESENDE，G.M.; MOURA, M.S.B.; DIAS, R.C.S.; COSTA, N.D.; CALGARO, M.; CORREIA, J.S.; SILVA, F.Z. Produtividade e qualidade do melão submetido a diferentes tipos de cobertura do solo. IRRIGA, n. 15, p. 422430, 2010.

COELHO, M.E.H. Manejo de plantas daninhas sobre a temperatura do solo, eficiência do uso da águia e crescimento do pimentão cultivado nos sistemas de plantio direto e convencional. Universidade Federal do semi-árido. Tese de doutorado. Mossoró, RN, 2011.

COELHO, M. E. H.; FREITAS, F. C. L de; CUNHA, J. L. X. L.; DOMBROSKI, J. L. D. SANTANA, F. A. O. de. Interferência de plantas daninhas no crescimento dopimen-tão nos sistemas de plantio direto e convencional. Revista Caatinga, v. 26, n.4, p. 19-30, 2013.

CONSTANTIN J. Métodos de manejo. In: OLIVEIRA JUNIOR RS; CONSTANTIN J. 
(eds). Plantas daninhas e seu manejo. Guaíba: Agropecuária, 2001. p.103-121.

CUDNEY, D. Nutsedge: history, economy, importance and distribution. In: NUTSEDGE MANAGEMENT WORKSHOP, n., 1997, Riverside: University of California, 1997. p. 2-3.

DURIGAN, J.C.; CORREIA, N. M.; TIMOSSI, P. C. Estádios de desenvolvimento e vias de contato e absorção dos herbicidas na inviabilização de tubérculos de Cyperus rotundus. Planta Daninha, v. 23, n. 4, p. 621-6, 2005.

ECHER, M.M.; FERNANDES, M.C.A.; RIBEIRO, R.L.D.; PERACCHI, A.L. Avaliação de genótipos de Capsicum para resistência ao ácaro branco. Horticultura Brasileira, v, 20, p. 217-221, 2002.

EMBRAPA - Empresa Brasileira de Pesquisa Agropecuária. Empresa Brasileira de Pesquisa Agropecuária Centro Nacional de Pesquisa de Solos. Sistema Brasileiro de Classificação de Solos. Brasília: Produções de Informações, 1999.

ERASMO, E. A. L.; AZEVEDO, W. R.; SARMENTO, R. A.; CUNHA, A. M.; GARCIA, S. L. R. Potencial de espécies utilizadas como adubo verde no manejo integrado de plantas daninhas. Planta Daninha, v. 22, p. 337-342, 2004.

GOMES JR., F. G.; CHRISTOFFOLETI, P. J. Biologia e manejo de plantas daninhas em áreas de plantio direto. Planta Daninha, v. 26, n. 4, p. 789-798, 2008.

LORENZI, H. Manual de identificação e controle de plantas daninhas: plantio e convencional. 6 ed. São Paulo: Instituto Plantarum, 2006. 339p.

PEREIRA, W; MELO, WF. Manejo de plantas espontâneas no sistema de produção orgânica de hortaliças. Boletim Técnico, 62. Brasilia: EMBRAPA Empresa Brasileira de Pesquisa Agropecuária., 2008. 